\title{
Decreased Susceptibility of Heart Mitochondria from Diabetic GK Rats to Mitochondrial Permeability Transition Induced by Calcium Phosphate
}

\author{
Paulo J. Oliveira, ${ }^{1}$ Anabela P. Rolo, ${ }^{1}$ R. Seiça, ${ }^{2}$ Carlos M. Palmeira, ${ }^{1}$ \\ M. S. Santos, ${ }^{1}$ and António J. M. Moreno ${ }^{1,3^{3}}$
}

Received October 6, 2000

\begin{abstract}
Type 2 diabetes (or non-insulin dependent diabetes mellitus, NIDDM) is a common metabolic disease in man. The Goto-Kakizaki (GK) rat has been designed as a NIDDM model. Previous studies with this strain have shown differences at the mitochondrial level. The mitochondrial permeability transition (MPT) is a widely studied phenomenon but yet poorly understood, that leads to mitochondrial dysfunction and cell death. The aim of this work was to compare the differences in susceptibility of induction of the MPT with calcium phosphate in GK and Wistar rats. Our results show that heart mitochondria from GK rats are less susceptible to the induction of MPT, and show a larger calcium accumulation before the overall loss of mitochondrial impermeability.
\end{abstract}

KEY WORDS: Type 2 diabetes; Goto-Kakizaki rat; mitochondrial permeability transition; heart mitochondria.

ABBREVIATIONS: $\Delta \Psi$, mitochondrial electric potential; BSA, bovine serum albumin; $\mathrm{TPP}^{+}$, tetraphenylphosphonium cation; MPT(P), mitochondrial permeability transition (pore); GK, Goto-Kakizaki; NIDDM, non-insulin dependent diabetes mellitus.

\section{INTRODUCTION}

Diabetes mellitus is a metabolic disorder characterized by high blood glucose levels. Two general types of diabetes mellitus are recognized. Type 1 patients are totally dependent on exogenous insulin, while type 2 patients have not such a dependence, being treated with dietary changes, exercise and some oral medication. With both types, numerous complications are common, including peripheral vascular disease, renal failure, coronary heart disease, blindness, infections and atherosclerosis [1].

\footnotetext{
${ }^{1}$ Centro de Neurociências de Coimbra, Dep. Zoologia, Faculdade de Ciências e Tecnologia, Universidade de Coimbra, Portugal.

${ }^{2}$ Faculdade de Medicina, Centro de Neurociências de Coimbra de Coimbra, Universidade de Coimbra, Portugal.

${ }^{3}$ To whom correspondence should be addressed. Fax: 351-239-826798; E-mail: Moreno@ci.uc.pt
} 
Type 2 (non-insulin dependent diabetes mellitus-NIDDM) is one of the most common metabolic diseases in man. The development of model animals carrying this pathology is of crucial importance to study the biochemical changes observed in the organism. The Goto-Kakizaki (GK) rat has been designed as a NIDDM model $[2,3]$ and used in several metabolic studies concerning that pathology.

Previous studies with GK rats showed several alterations in mitochondrial bioenergetics $[4,5]$ and mitochondrial antioxidant capacity [6], suggesting specific changes in that organelle. Further studies are thus necessary to fully enumerate the biochemical changes observed at the mitochondrial level, that may contribute to the overall effect observed in a specific organ in NIDDM. An aspect that has still not been studied in this type of diabetes is the susceptibility of diabetic GK rats to the induction of mitochondrial permeability transition (MPT) (for reviews, see [7-10]). The MPT is thought to envolve the formation of a proteinaceous pore (the MPTP), induced by several agents. The opening of the MPTP causes a sudden increase in membrane permeability, leading to mitochondrial swelling, membrane potential depolarization, calcium release and a burst of mitochondrial oxygen consumption, among other effects.

The aim of this work was to study the susceptibility of induction of MPT induced by calcium phosphate on heart mitochondria isolated from GK and Wistar rats. Our results clearly show that heart mitochondria isolated from GK rats are more resistant to that phenomenon, in comparison with Wistar rats of the same age (26 weeks), which interfere with the normal calcium pathways of the cell.

\section{MATERIALS AND METHODS}

\section{Animals}

Male GK rats (26 weeks) were obtained from our colony with breeding couples from the colony at Tohoku University School of Medicine (Sendai, Japan, courtesy of Dr. K. I. Susuki). Control animals were normal male Wistar rats (26 weeks). GK rats showed no fasting hyperglycemia $(277.4 \pm 15.8 \mathrm{mg} / \mathrm{dl})$ in comparison with Wistar rats $(105.4 \pm 3.0 \mathrm{mg} / \mathrm{dl})$.

\section{Materials}

All reagents and chemicals used were of the highest grade of purity commercially available. Calcium Green 5-N was obtained from Molecular Probes, Eugene, OR.

\section{Preparation of Heart Mitochondria}

Rat heart mitochondria were prepared using a conventional procedure. Briefly, the rats were killed by cervical dislocation and the hearts were immediately excised and minced finely in an ice-cold isolation medium containing $250 \mathrm{mM}$ sucrose, $1 \mathrm{mM}$ EGTA, $10 \mathrm{mM}$ Hepes-KOH (pH 7.4) and 0.1\% defatted BSA. The minced bloodfree tissue was then resuspended in $40 \mathrm{ml}$ of isolation medium containing $1 \mathrm{mg}$ protease Type VIII (Sigma no. P-5390) per mg of tissue and homogenized with a tightly 
fitted homogenizer (Teflon:glass pestle). The suspension was incubated for $1 \mathrm{~min}$ $\left(4^{\circ} \mathrm{C}\right)$ and then re-homogenized. The homogenate was then centrifuged at $10,000 \mathrm{~g}$ for $10 \mathrm{~min}$ (Sorvall RC-5C, Plus, SS 34 rotor, $4^{\circ} \mathrm{C}$ ). The supernatant fluid was decanted and the pellet, essentially devoid of protease, was gently homogenized to its original volume with a loose-fitting homogenizer. The suspension was centrifuged at $500 \mathrm{~g}$ for $10 \mathrm{~min}$ and the resulting supernatant was centrifuged at $10,000 \mathrm{~g}$ for $10 \mathrm{~min}$. The pellet was resuspended using a paint brush and repelleted twice at $10,000 \mathrm{~g}$ for $10 \mathrm{~min}$, EGTA and defatted BSA were omitted from the final washing medium. Mitochondrial protein content was determined by the biuret method calibrated with BSA.

\section{Membrane Potential $(\Delta \Psi)$ Determinations}

The mitochondrial transmembrane potential $(\Delta \Psi)$ was estimated with a $\mathrm{TPP}^{+}$ electrode according to Kamo et al. [11] without correction for the "passive" binding contribution of $\mathrm{TPP}^{+}$to the mitochondrial membrane because the purpose of the experiments was to show relative changes in the potential rather than absolute values. A matrix volume of $1.1 \mu \mathrm{l} / \mathrm{mg}$ protein was assumed. Reactions were carried out at $25^{\circ} \mathrm{C}$ in $2 \mathrm{ml}$ of the standard medium $(200 \mathrm{mM}$ sucrose, $10 \mathrm{mM}$ Tris-Mops, $10 \mu \mathrm{M}$ EGTA, $5 \mathrm{mM} \mathrm{KH}_{2} \mathrm{PO}_{4}$, pH 7.4) supplemented with $3 \mu \mathrm{M} \mathrm{TPP}{ }^{+}$and $1 \mathrm{mg}$ of mitochondria. Energized mitochondria were obtained with $8 \mathrm{mM}$ succinate (plus $4 \mu \mathrm{M}$ rotenone and $0.5 \mu \mathrm{g}$ oligomycin).

\section{Determination of Mitochondrial Swelling}

Mitochondrial osmotic volume changes were followed by monitoring the classic decrease of absorbance at $540 \mathrm{~nm}$ with a Jasco V-560 spectrophotometer. The assays were performed in $2 \mathrm{ml}$ of medium $(200 \mathrm{mM}$ sucrose, $10 \mathrm{mM}$ Tris-Mops, $10 \mu \mathrm{M}$ EGTA, $5 \mathrm{mM} \mathrm{KH}_{2} \mathrm{PO}_{4}, 4 \mu \mathrm{M}$ rotenone, $0.5 \mu \mathrm{g}$ oligomycin and $8 \mathrm{mM}$ succinate) and with $1 \mathrm{mg}$ protein.

\section{Determination of Mitochondrial Oxygen Consumption}

Oxygen consumption of heart mitochondria from both GK and Wistar rats was monitored polarographically with a Clark oxygen electrode connected to a suitable recorder in a 1-ml thermostatted water-jacketed closed chamber with magnetic stirring, at $25^{\circ} \mathrm{C}$. The standard respiratory medium consisted of $200 \mathrm{mM}$ sucrose,

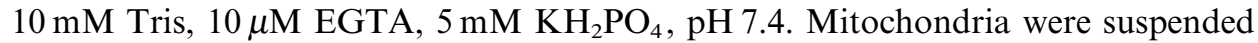
at a concentration of $1 \mathrm{mg} / \mathrm{ml}$ in the respiratory medium. State IV respiration was measured in the presence of $8 \mathrm{mM}$ succinate (plus $4 \mu \mathrm{M}$ rotenone).

\section{Measurement of Mitochondrial $\mathrm{Ca}^{2+}$ Uptake}

Extramitochondrial free $\mathrm{Ca}^{2+}$ was measured with the hexapotassium salt of the fluorescence probe Calcium Green 5-N [12]. Heart mitochondria (0.2 mg) from GK and Wistar rats were resuspended in $2 \mathrm{ml}$ of buffer containing $200 \mathrm{mM}$ sucrose, 


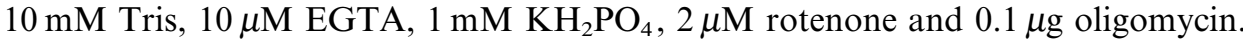
Free $\mathrm{Ca}^{2+}$ was monitored with $100 \mathrm{mM}$ Calcium Green 5-N. Fluorescence was recorded continuously in a water-jacketed cuvette holder at $25^{\circ} \mathrm{C}$ using a PerkinElmer LS-50B fluorescence spectrometer with excitation and emission wavelengths of 506 and $531 \mathrm{~nm}$, respectively. Energization was obtained with $8 \mathrm{mM}$ succinate. At the end of each experiment, excess EGTA was added to obtain the basal line. The calibration was made with known amounts of calcium.

\section{RESULTS}

The calcium accumulation capacity of heart mitochondria from Wistar and GK rats was determined observing the effect of known quantities of calcium in the mitochondrial $\Delta \Psi$, measured with a $\mathrm{TPP}^{+}$-selective electrode. Figure 1 shows that after the addition of $500 \mu \mathrm{M} \mathrm{Ca}^{2+}$, heart mitochondria from $\mathrm{GK}$ rats are capable of

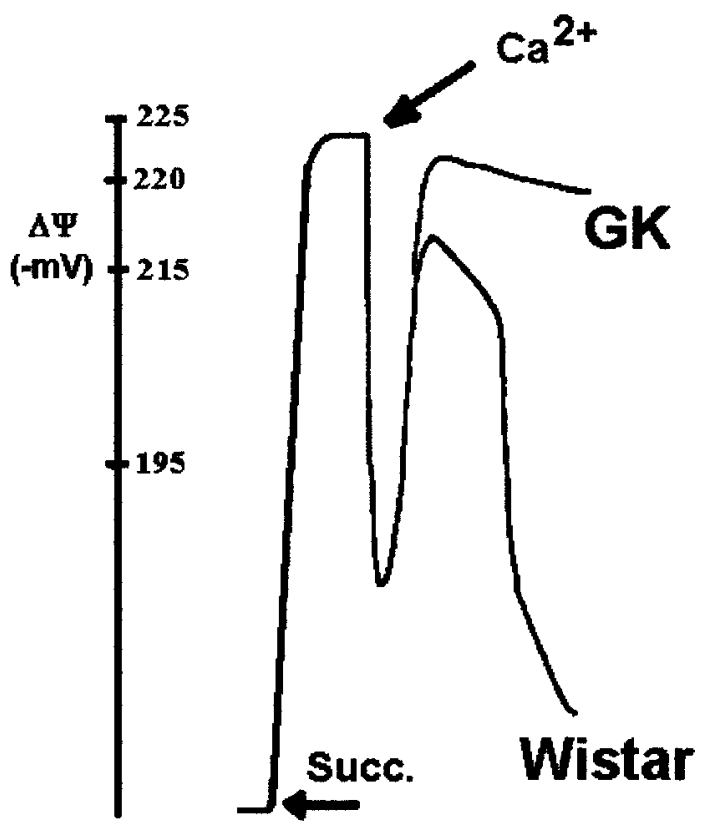

Fig. 1. Mitochondrial electric potential measurements with a $\mathrm{TPP}^{+}$-selective electrode. Calcium $(600 \mu \mathrm{M}$ soluble salt $)$ was added in order to induce the MPTP. The $\Delta \Psi$ was calculated without correction for $\mathrm{TPP}^{+}$-passive binding to mitochondrial membranes. $1 \mathrm{mg}$ mitochondrial protein was resuspended in $2 \mathrm{ml}$ reaction medium ( $200 \mathrm{mM}$ sucrose, $10 \mathrm{mM}$ Tris, $10 \mu \mathrm{M}$ EGTA and $5 \mathrm{mM} \mathrm{KH}_{2} \mathrm{PO}_{4}$, supplemented with $4 \mu \mathrm{M}$ rotenone, $0.5 \mu \mathrm{g}$ oligomycin and $3 \mu \mathrm{M}$ $\mathrm{TPP}^{+}$). Mitochondrial energization was achieved with $8 \mathrm{mM}$ succinate. The traces are representative of five different preparations. 
maintaining the $\Delta \Psi$ at its original value, a feature not shared by its Wistar counterparts. In the latter case, a sudden loss of $\Delta \Psi$ was observed, compatible with opening of the MPTP (and inhibited by cyclosporin, not shown). The same result was observed when we tested the effect of calcium pulses of lower concentrations. Mitochondria from GK rats could handle a larger number of pulses than mitochondria from Wistar rats (not shown).

Concomitant with these observations, two other events that revealed the opening of the MPTP were studied, namely mitochondrial swelling and oxygen consumption. It is widely known that the opening of the MPTP induces mitochondrial swelling and a stimulated mitochondrial oxygen consumption. So, we tested both situations and indeed, mitochondria from GK rats not only showed a smaller amplitude of swelling after the addition of $600 \mu \mathrm{M}$ of calcium (Fig. 2), but it was also possible to observe differences in mitochondrial oxygen consumption. In Fig. 2 (inset), we can see that mitochondrial respiration after calcium addition $(600 \mu \mathrm{M})$ in Wistar strain does not recover the normal state IV values, showing that the inner membrane permeability to protons has increased. In another set of experiments, the addition of lower concentrations of calcium (with full recovery of state IV respiration), unequivocally showed that the parameter $\mathrm{Ca}^{2+} / \mathrm{O}$, that can tell us the quantity of oxygen consumed during full accumulation of added calcium, was always bigger in mitochondria isolated from GK rats than from Wistar strain (data not

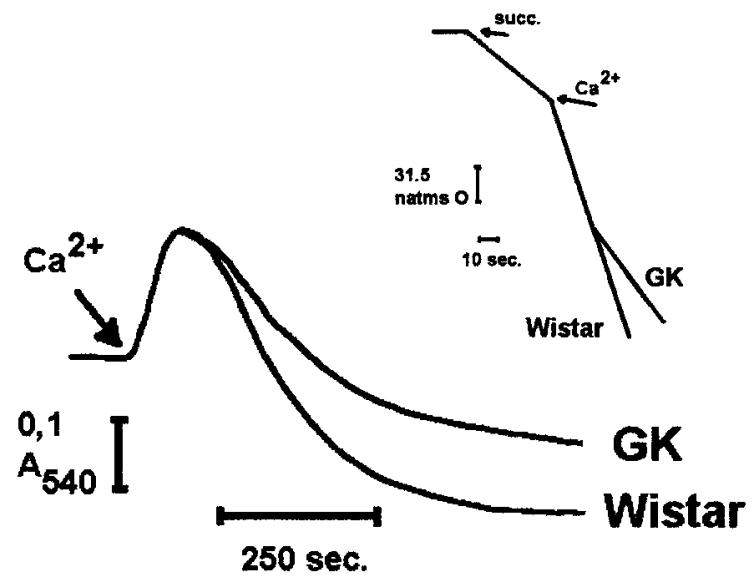

Fig. 2. Typical recording of mitochondrial swelling (decrease of absorbance at $540 \mathrm{~nm})$ induced by calcium $(600 \mu \mathrm{M}$ soluble salt). Heart mitochondria isolated from both GK and Wistar rats $(0.5 \mathrm{mg} / \mathrm{ml})$ were incubated in $2 \mathrm{ml}$ of media constituted by $200 \mathrm{mM}$ sucrose, $10 \mathrm{mM}$ Tris-Mops, $10 \mu \mathrm{M}$ EGTA and $5 \mathrm{mM}$ $\mathrm{KH}_{2} \mathrm{PO}_{4}$, supplemented with $4 \mu \mathrm{M}$ rotenone, $0.5 \mu \mathrm{g}$ oligomycin and $8 \mathrm{mM}$ succinate. After establishing a basal line, calcium was added. Inserted is a typical recording of mitochondrial oxygen consumption after the addition of calcium $(600 \mu \mathrm{M})$ under the same conditions. State IV respiration was induced with $8 \mathrm{mM}$ succinate. The traces are representative of three different preparations. 


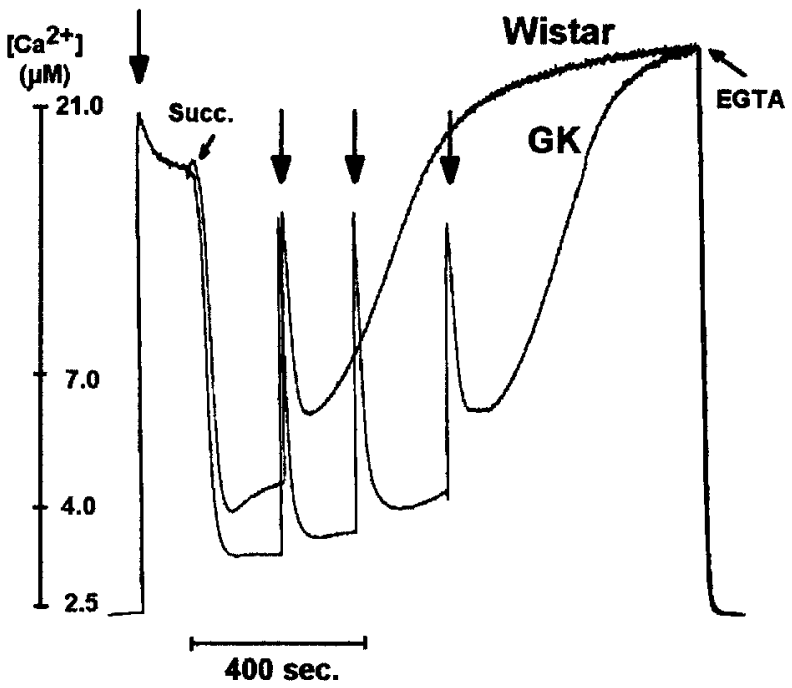

Fig. 3. Typical measurements of extramitochondrial calcium movements using the fluorescent calcium-sensitive probe Calcium Green 5-N. The ordinate quantifies the extramitochondrial calcium and bold arrows show calcium additions. EGTA was added in the end of the experiments to calculate the basal line. Mitochondria $(0.2 \mathrm{mg})$ were incubated in $2 \mathrm{ml}$ of reaction medium containing $200 \mathrm{mM}$ sucrose, $10 \mathrm{mM}$ Tris-Mops, $10 \mu \mathrm{M}$ EGTA and $5 \mathrm{mM}$ $\mathrm{KH}_{2} \mathrm{PO}_{4}$, supplemented with $2 \mu \mathrm{M}$ rotenone, $0.1 \mu$ oligomycin. Mitochondrial energization was achieved with $4 \mathrm{mM}$ succinate. The traces are representative of three different preparations.

shown), which is indicative that Wistar mitochondria must consume a larger quantity of oxygen (and thus take more time) to accumulate the same quantity of calcium.

The final evidence comes from determinations of extramitochondrial calcium movements using the fluorescent calcium-sensitive probe Calcium Green 5-N. The results (Fig. 3) again confirm the previous data. Mitochondria from GK rats were able to accumulate a larger quantity of calcium than mitochondria from Wistar rats. Cyclosporin A was able to inhibit calcium release, thus confirming the opening of the MPTP. The same results were obtained when a larger single calcium pulse was given: heart mitochondria from GK rats were able to retain calcium longer than those from Wistar rats.

\section{DISCUSSION}

Diabetes is a complex and costly disease that can affect nearly every organ in the body and result in devastating consequences. Diabetes can cause nontraumatic lower extremity amputations, renal failure, blindness, premature mortality, stroke, cardiovascular disease, peripheral vascular disease, congenital malformations, among others [13]. Among the two recognized types of diabetes, type 2 diabetes mellitus (non-insulin-dependent diabetes mellitus-NIDDM) is the most common. 
It is a metabolic disorder characterized by hyperglycemia without absolute insulin deficiency. Due to the prevalence of this pathology and the complications that may arise for the patient, it is necessary to characterize all the steps of the disease, either in human patients or in animal models. The Goto-Kakizaki is an inbred rat strain developed by selective breeding with glucose intolerance as a selection index of an outbred colony of nondiabetic Wistar strain rats, being a well-characterized model for non-obese type 2 diabetes [2,3]. Changes in mitochondrial bioenergetics and antioxidant defenses were characterized [4-6], showing for example, that liver mitochondrial preparations isolated from GK rats (6 months old) have a decreased susceptibility to lipid peroxidation induced by $\mathrm{ADP} / \mathrm{Fe}^{2+}$, when compared to a Wistar strain [6].

Mitochondria and diabetes (NIDDM in this case) are two faces of the same coin. Mitochondria can be one of the causes of the pathology, or they can be affected by the course of the disease. Different types of mitochondrial DNA mutations are associated with diabetes (reviewed in [14]), which is understandable if we recall that mitochondrially-produced ATP is probably the main intermediate between glucose sensing and insulin secretion in pancreatic $\beta$-cells (see for example $[15,16]$ ). Mitochondria can be affected by the increased oxidative stress load observed in the course of the disease (for a review of the role of oxidative stress in the development of complications in diabetes, see [17]). With this in mind, the GK rats provide an excellent opportunity to study all the mitochondrial alterations associated with NIDDM.

Not yet studied in this model of NIDDM is the induction of the mitochondrial permeability transition with calcium phosphate. The MPT has been attributed to the opening of a proteic pore, located in the inner mitochondrial membrane, with a cut-off of $1500 \mathrm{KDa}$, that leads to a loss of membrane impermeability, with concomitant membrane depolarization, calcium release, mitochondrial swelling and a burst of mitochondrial respiration (for reviews see [7-10]). From this starting point, we isolated mitochondria from an organ widely affected in this pathology, namely the heart, $[18,19]$ from both Wistar and GK rats.

From our results we can say, without doubt, that heart mitochondria from GK rats are less susceptible to MPT induced by calcium phosphate, achieved by adding soluble $\mathrm{Ca}^{2+}$ to phosphate-containing medium. These results are seen in Figs. 1-3. Measurements of mitochondrial $\Delta \Psi$ in GK vs. Wistar rats (Fig. 1) showed that the former were able to maintain $\Delta \Psi$ after the addition of calcium, in contrast with Wistar ones, where a drop in that parameter is clearly seen, in a cyclosporin Ainhibitable manner (not shown). In Fig. 2, two different parameters are seen, mitochondrial swelling and oxygen consumption. Again, mitochondria from GK rats show a lesser susceptibility to the addition of calcium, suffering a smaller swelling and being capable of recovering oxygen consumption to state IV values. This last result is confirmed by measurements of the parameter $\mathrm{Ca}^{2+} / \mathrm{O}$, in which mitochondria from GK rats showed increased values, indicative of a faster accumulation and lesser susceptibility to calcium-induced calcium release.

Following the extramitochondrial calcium movements led us to the same conclusions. Heart mitochondria from GK rats showed a larger propensity to accumulate calcium, that is, GK mitochondria showed a delayed release of the accumulated calcium (again, in a cyclosporin A-inhibitable manner (not shown)). 
Our results are in agreement with previous observations on the delay of MPT in liver mitochondria from streptozotocin-induced diabetes (type 1) in SpragueDawley rats [20], showing that this resistance to calcium phosphate-induced MPTP is shared by both types of diabetes.

The precise mechanisms for these differences are, for now, unknown but may involve some up-regulation of the antioxidant defense mechanisms in mitochondria (suggested in [6]), opposed to the increased oxidative stress associated to this pathology and indeed observed in GK rats [21]. Another hypothesis that is being tested in our laboratory which seems attractive involves a possible reduction in the levels of heart hexokinase bound to mitochondria that may, indeed, contribute to these results, as hexokinase has been involved in the MPTP complex [22]. Nevertheless, the lack of hexokinase in the liver does not support this hypothesis as this difference was also observed in liver mitochondria isolated from rats with diabetes type I [20].

Whatever the mechanism (or mechanisms) is, the increased capacity of heart mitochondria from GK rats to accumulate calcium may contribute to the observed resistance of diabetic hearts to ischemia-reperfusion injury, as the MPTP has been associated with that condition [23] and to alterations in the normal cellular calcium pathways.

In conclusion, heart mitochondria from GK rats displayed an increased resistance to the MPT induced by calcium phosphate, suggesting that this feature is common to both type of diabetes and may include mitochondria from different sources.

\section{ACKNOWLEDGMENTS}

We wish to acknowledge financial support from the Portuguese Foundation for Science and Technology-Research Project PRAXIS/PSAU/S/16/96. P.O. and A.R. are supported by Ph.D. grants from the Portuguese Foundation for Science and Technology (PRAXIS XXI/BD/21494/99 and PRAXIS XXI/BD/21454/99, respectively). The authors wish to thank Dario L. Santos, Vilma A. Sardão and Pedro M. Coxito for their assistance in this work.

\section{REFERENCES}

1. Flatt, P., Abdel-Wahab, Y., Boyd, A. C. et al. (1997) Proc. Nutr. Soc. 56:243-262.

2. Kimura, K., Toyoto, T., Kakizaki, M., Kudo, M., Takebe, K., and Goto, Y. (1982) Tohoku J. Exp. Med. 137:453-459.

3. Goto, Y., Kakizaki, M., and Masaki, N. (1975) Proc. Jpn. Acad. 51:80-85.

4. Ferreira, F. M. L., Palmeira, C. M., Seiça, R., and Santos, M. S. (1999) Metabolism 48:1115-1119.

5. Palmeira, C. M., Ferreira, F. M. L., Santos, D. L., Ceiça, R., Suzuki, K.-i., and Santos, M. S. (1999) FEBS Lett. 458:103-106.

6. Ferreira, F. M. L., Palmeira, C. M., Matos, M. J., Seiça, R., and Santos, M. S. (1999) Life Sci. 65:1013-1025.

7. Zoratti, M. and Szabò, I. (1995) Biochim. Biophy. Acta 1241:139-176.

8. Vercesi, A. E., Kowaltowski, A. J., Grijalba, M. T., Meinicke, A. R., and Castilho, R. F. (1997) Biosc. Rep. 17:43-52.

9. Crompton, M. (1999). Biochem. J. 341: 233-249.

10. Bernardi, P. (1999) Physiol. Rev. 79:1127-1155.

11. Kamo, N., Muratsugu, M., Hongoh, R., and Kobatake, Y. (1979) J. Membrane Biol. 49:105-121. 
12. Rajdev, S. and Reynolds, I. J. (1993) Neurosc. Lett. 162:149-152.

13. Engelgau, M. and Geiss, L. S. (2000) In: The Burden of Diabetes Mellitus (J. L. Leahy, N. G. Clark, and W. T. Cefalu, eds.), Medical Management of Diabetes Mellitus, Marcel Dekker, New York.

14. Gerbitz, K.-D., Van den Ouweland, J. M. W., Maassen, J. A., and Jaksch, M. (1995) Biochem. Biophys. Acta 1271:253-260.

15. Anderson, C. M. (1999) Drug Dev. Res. 46:67-79.

16. Gerbitz, K.-D., Gempel, K., and Brdiczka, D. (1996) Diabetes 45:113-126.

17. Baynes, J. W. (1991) Diabetes 40:405-412.

18. Leinonen, J. et al. (1998) Free Rad. Res. 29:273-281.

19. King, G. L. and Wakasaki, H. (1999) Diabetes Care 22:C31-C37.

20. Kristal, B. S., Matsuda, M., and Yu, B. P. (1996) Biochem. Biophys. Res. Commun. 222:519-523.

21. Ihara, Y. et al. (1999) Diabetes 48:927-932.

22. Beutner, G., Ruck, A., Riede, B., and Brdiczka, D. (1998) Biochim. Biophys. Acta 1368:7-18.

23. Gunter, T. T., Gunter, K. K., Sheu, S.-S., and Gavin, C. E. (1994) Am. J. Physiol. 267:C313-C339. 